\title{
Aceitação e perfil sensorial das cachaças produzidas com Kefir e Saccharomyces cerevisae
}

\author{
Acceptance and sensory profile of cachaça produced using Kefir and Saccharomyces cerevisae
}

Anita Saraiva DORNELLES ${ }^{1}$, Sueli RODRIGUES ${ }^{2 *}$, Deborah dos Santos GARRUTI ${ }^{3}$

\section{Resumo}

A levedura Saccharomyces cerevisae é o microrganismo mais utilizado industrialmente nas destilarias, mas outros microrganismos também são capazes de produzir etanol utilizando matérias-primas açucaradas como substrato. O objetivo deste trabalho foi determinar o perfil sensorial e a aceitação da aguardente de cana produzida através da fermentação alcoólica com grânulos de Kefir, comparando-a ao produto obtido tradicionalmente. Pelo método de ADQ, 8 provadores treinados avaliaram os seguintes descritores: aroma alcoólico e de cachaça, sabor alcoólico e de cachaça, gosto amargo e ardência. O teste de aceitação foi realizado por 57 consumidores de aguardente, utilizando-se uma escala hedônica estruturada de nove pontos para avaliar a aceitação do aroma e a aceitação global. A cachaça com Kefir apresentou maior intensidade de aroma alcoólico e gosto amargo, obtendo menor aceitação global que a cachaça de levedura. Dessa forma, o produto foi considerado de grande potencial visto que apresentou satisfatório percentual de aprovação entre os consumidores.

Palavras-chave: cachaça; fermentação alcoólica; análise sensorial; análise descritiva quantitativa.

\begin{abstract}
Saccharomyces cerevisae is the main industrially used yeast to produce sugar cane spirits (cachaça). However, other microorganisms are able to produce ethanol using sugar as substrate. This work aimed at determining the sensory profile of cachaça produced through the alcoholic fermentation with Kefir's granules and its acceptance. The acceptance analysis was performed by 57 consumers using a 9 point structured hedonic scale to evaluate the aroma and the general acceptance of the product. The QDA method, with 8 trained tasters, was used to evaluate the following product descriptors: alcoholic aroma, alcoholic flavor, and burning and bitter taste. The evaluated samples did not presented statistical differences $(\mathrm{p} \leq 0.05)$ among each other regarding the aroma acceptance. However, the cachaça produced using bakery yeast presented higher acceptance scores. On the other hand, the analysis of general acceptance presented significant differences $(p \leq 0.05)$ between the samples for alcoholic aroma and bitter taste. Cachaça produced with Kefir granules presented higher intensity of both of these attributes. Keywords: cachaça; alcoholic fermentation; sensory analysis; quantitative descriptive analysis.
\end{abstract}

\section{Introdução}

Definida, oficialmente, como a denominação típica e exclusiva da aguardente de cana produzida no Brasil, com graduação alcoólica de 38 a $48 \%$ em volume, a $20^{\circ} \mathrm{C}$ e com características sensoriais peculiares, a cachaça, com sua origem datando do século XVII, quando a cana-de-açúcar começou a ser plantada na Região Nordeste em meio à colonização portuguesa, é bebida tradicional do Brasil. Da mesma maneira, acontece em outros países do mundo, onde também existem bebidas que, em muitos casos, chegam até mesmo a fazer parte das suas tradições (AQUINO et al., 2006). Com seu sabor característico, as cachaças vêm conquistando novos mercados nacionais e nos últimos anos apresentou um crescimento perceptível no mercado internacional. Dessa forma estudos inovadores são de suma importância.

O principal processo produtivo de aguardente é realizado em batelada e utiliza matérias-primas açucaradas, tais como melaço e caldo de cana, e como agente fermentador, a levedura (Saccharomyces cerevisae). Entretanto, outros microrganismos são também capazes de produzir etanol utilizando matériasprimas açucaradas como substrato (DORNELLES; RODRIGUES, 2006).

Os grânulos de Kefir são formados, na maior parte, de proteínas e polissacarídeos em que uma complexa microbiota é incluída. Os grânulos de Kefir são constituídos por água (890-900 g/kg), lipídios $(2 \mathrm{~g} / \mathrm{kg})$, proteínas $(30 \mathrm{~g} / \mathrm{kg})$, açúcares $(60 \mathrm{~g} / \mathrm{kg})$ e minerais $(7 \mathrm{~g} / \mathrm{kg})$ (GARROTE et al., 2006). A bebida fermentada à base de leite produzida com grânulos de Kefir é frequentemente associada à longevidade, sendo considerada terapêutica. Esses grânulos também são capazes de produzir compostos de aroma e sabor durante a fermentação, tornando os produtos obtidos através de fermentação com Kefir diferenciados.

\footnotetext{
Recebido para publicação em 24/10/2007

Aceito para publicação em 17/7/2008 (002936)

${ }^{1}$ Laboratório de Biotecnologia, Departamento de Tecnologia de Alimentos, Universidade Federal do Ceará - UFC, Fortaleza - CE, Brasil,

E-mail: anitasaraivadornelles@yahoo.com.br

2 Centro de Ciências Agrárias, Departamento de Tecnologia de Alimentos, Universidade Federal do Ceará - UFC, Campus do Pici, s/n, CP12168, CEP 60356-000,

Fortaleza-CE, E-mail: sueli@ufc.br

${ }^{3}$ Embrapa Agroindustrial Tropical, Laboratório de Análise Sensorial, Fortaleza - CE, E-mail: deborah@cnpat.embrapa.br

${ }^{*}$ A quem a correspondência deve ser enviada
} 
Apesar da importância econômica e social da cachaça, são ainda escassos os estudos sobre sua qualidade sensorial, porém as crescentes exigências do mercado têm aumentado a preocupação com a qualidade dessa bebida (CARDELLO; FARIA, 1998; JANZANTTI, 2004).

Boza e Horri (1998) investigaram o comportamento das substâncias importantes para a qualidade sensorial da aguardente de cana de açúcar durante o processo de destilação e constataram a necessidade de ampliar o número de componentes analisados das amostras de aguardentes, para aumentar o coeficiente de correlação entre os dados analíticos e sensoriais.

A influência da concentração alcoólica e da acidez na percepção sensorial de aguardente de cana foi estudada e concluiu-se que esses parâmetros não influíram na avaliação sensorial dos atributos típicos da aguardente (JERÔNIMO; CARDELLO; SERRA, 2004).

O presente trabalho teve como objetivos traçar o perfil da cachaça produzida com grânulos de Kefir através da análise descritiva comparando-o com o perfil de uma cachaça produzida com S. cerevisae e avaliar a aceitabilidade dessas amostras frente a um produto comercial líder de mercado.

\section{Material e métodos}

\subsection{Amostras}

Os grânulos de kefir utilizados neste trabalho são pertencentes ao Laboratório de Biotecnologia (LABIOTEC/ DETAL/UFC). Já a Saccharomyces cerevisae (fermento biológico seco instantâneo Fleischmann) e o caldo de cana foram obtidos junto ao comércio local.

As cachaças de Kefir e de S. cerevisae foram produzidas no laboratório de Biotecnologia utilizando-se as condições de melhor rendimento em etanol determinadas em um estudo anterior a partir de um planejamento fatorial de ensaios de fermentação com os grânulos de Kefir e a levedura S. cerevisae variando-se a quantidade de inóculo e a concentração de sacarose (DORNELLES et al., 2006).

Foi realizada uma fermentação em larga escala utilizando os grânulos de Kefir e outra utilizando a levedura S. cerevisae. Foram fermentados $25 \mathrm{~L}$ de caldo de cana com grânulos de Kefir e $25 \mathrm{~L}$ com levedura S. cerevisae. Nos dois casos, foi ajustado o Brix do caldo de cana para 19 (DORNELLES et al., 2006). Na fermentação com grânulos de Kefir, inicialmente, utilizaram-se 30 g.L. $\mathrm{L}^{-1}$ de levedura em base seca para produção da invertase. Após 40 minutos, foi feita a remoção das células por centrifugação durante 10 minutos 11806 g. Foram então adicionadas 300 g.L. $\mathrm{L}^{-1}$ de grânulos de Kefir em base úmida. A fermentação foi então conduzida por 24 horas em temperatura ambiente $\left(30^{\circ} \mathrm{C}\right)$. As células de Kefir foram então removidas por peneiramento.

$\mathrm{Na}$ fermentação com levedura S. cerevisae utilizou-se uma biomassa de 20 g.L.- (fermento seco) e, após 24 horas, as células foram removidas por centrifugação a $11806 \mathrm{~g}$ por 10 minutos.
A destilação foi realizada através de um destilador artesanal de aço inox com $30 \mathrm{~L}$ de capacidade, composto por duas partes: a parte inferior (caldeira), que aquece o caldo fermentado através de uma resistência; e a parte superior, um condensador refrigerado à água. A destilação foi conduzida a $80^{\circ} \mathrm{C}$, sendo recolhidas as frações cabeça, calda e coração de acordo com o teor alcoólico do destilado. A cabeça e a cauda foram separadas e o coração foi aproveitado. As duas cachaças foram submetidas às análises físico-químicas de teor alcoólico, acidez total, acidez volátil, ésteres (em acetato de etila), aldeídos (em aldeído acético), cobre, álcoois superiores e metanol (DORNELLES, 2007).

\subsection{Análise sensorial descritiva}

O perfil sensorial das amostras de cachaça foi determinado utilizando-se a técnica de Análise Descritiva Quantitativa (ADQ) (STONE et al., 1974). Os testes foram realizados no Laboratório de Análise Sensorial da Embrapa Agroindústria Tropical, em Fortaleza - CE, cujas instalações incluem cabines individuais e controle de iluminação e temperatura ambiente $\left(28 \pm 2{ }^{\circ} \mathrm{C}\right)$.

\section{Recrutamento dos provadores}

Foram recrutados 15 indivíduos dentre os estagiários, pesquisadores e funcionários da Embrapa Agroindústria Tropical. Os critérios foram: ser consumidor de aguardente e ter sido selecionado anteriormente para participar em equipes de análise sensorial descritiva de outros produtos, passando por testes de seleção quanto à sua acuidade sensorial normal (reconhecimento de aromas e gostos básicos).

\section{Desenvolvimento da terminologia descritiva}

Para o levantamento de termos que descrevessem o perfil das cachaças, foram escolhidas quatro amostras de cachaças comerciais que apresentavam diferenças marcantes de sabor. As cachaças foram servidas em taças de vidro com tampa, codificadas com números de três dígitos, contendo cerca de $20 \mathrm{~mL}$ da amostra, sendo solicitado que cada julgador descrevesse as características da amostra em relação ao sabor e ao aroma. Em seguida foi elaborada uma listagem de todos os termos gerados e o número de vezes que eles foram citados e conduzidas discussões em grupo com o objetivo de gerar os termos descritivos semelhantes, definições e amostras de referência dos extremos da escala de intensidade. Sessões suplementares de avaliação das amostras, das referências e de discussão em grupo resultaram na ficha final de avaliação das amostras.

\section{Seleção de provadores e análise descritiva das amostras}

As cachaças com Kefir e com levedura foram analisadas pela equipe inicial de 15 julgadores, em triplicata, em três sessões em dias consecutivos. Foi utilizada a ficha de avaliação desenvolvida em consenso com a equipe. Nessa ficha, cada termo descritivo era avaliado em uma escala não estruturada de $9 \mathrm{~cm}$, ancorada nos extremos com os seguintes termos de intensidade: fraco e 
forte para aroma alcoólico, aroma de cana, sabor alcoólico, sabor de cana, ardência inicial e ardência residual; nenhum e forte para gosto doce e gosto amargo. As cachaças foram servidas monadicamente, em taças de vidro com tampa, codificadas com números aleatórios de três dígitos, contendo cerca de $20 \mathrm{~mL}$ da amostra, acompanhadas de um copo de água e um pedaço de pão de fôrma para limpar o paladar entre as amostras. Os testes foram realizados em cabines individuais. Foi realizada uma análise de variância (ANOVA) para os resultados de cada provador, para cada descritor avaliado, tendo como fontes de variação amostras e repetições. Foram selecionados, para compor a equipe descritiva final, aqueles provadores que apresentaram bom poder discriminativo $\left(\mathrm{p}_{\text {amostra }} \leq 0,50\right)$, boa reprodutibilidade nos julgamentos $\left(\mathrm{p}_{\text {repeticão }} \geq 0,05\right)$ e consenso com os demais membros do grupo, avaliado pelo gráfico das médias das amostras para cada julgador. Assim, a equipe ficou formada por 8 (oito) provadores. Em seguida, os dados da equipe final foram submetidos à análise de variância tendo como fontes de variação amostras, provadores e a interação entre eles. As análises estatísticas foram realizadas com o auxílio do pacote estatístico SAS for Windows, utilizando-se a rotina GLM (General Linear Models) e o próprio teste $F$ para comparação das médias.

\subsection{Teste de aceitação}

Os testes de aceitação foram realizados segundo metodologia descrita por Meilgaard, Civille e Carr (1987), no Laboratório de Análise Sensorial da Embrapa Agroindústria Tropical, em Fortaleza - CE, com 57 indivíduos, sendo 54,4\% do sexo masculino e $45,6 \%$ do sexo feminino. Dentre os provadores, $60 \%$ estavam na faixa etária de 18 a 25 anos de idade, e 33\% entre 26 e 35 anos, caracterizando-se como um público adulto jovem e que consomem aguardente frequentemente.

As amostras analisadas foram: cachaça obtida com Kefir, cachaça obtida com levedura e uma cachaça comercial líder de mercado e não envelhecida, com teor alcoólico de 39\% (v/v). A cachaça comercial foi utilizada para comparação da aceitação. As amostras foram servidas monadicamente, em taças de vidro com tampa, codificadas com números aleatórios de três dígitos, contendo cerca de $20 \mathrm{~mL}$ da amostra, acompanhadas de um copo de água e um pedaço de pão de fôrma para limpar o paladar entre as amostras. O teste foi realizado em uma única etapa, sendo que o provador tinha um intervalo de 10 minutos entre uma amostra e outra. Os testes foram realizados em cabines individuais.

Para a avaliação do grau de aceitação do aroma e aceitação global, foi utilizada uma escala hedônica estruturada de 9 pontos, variando entre "desgostei muitíssimo" até "gostei muitíssimo", perguntando-se ao provador o que ele mais gostou e menos gostou nas amostras. As categorias da escala hedônica atribuídas pelos consumidores a cada amostra foram posteriormente convertidas a valores numéricos, para o tratamento estatístico, sendo 1 = desgostei muitíssimo e 9 = gostei muitíssimo.

Os valores hedônicos foram avaliados através da análise de variância (ANOVA), tendo como fontes de variação: amostra (A), provador (P) e teste de Tukey para comparação das médias. O programa utilizado foi o Statistical Analytical Systems (SAS, 2000).

\section{Resultados e discussão}

Após ter sido obtida a lista de termos descritivos, chegouse, por consenso, a uma lista com 7 (sete) descritores que caracterizaram sensorialmente as amostras de cachaças elaboradas. A Tabela 1 apresenta cada descritor com a respectiva definição e amostras de referência.

Os resultados da Análise de Variância da intensidade de cada descritor, para as duas cachaças avaliadas (produzidas com Kefir e com levedura), são apresentados na (Tabela 2). Observou-se que as amostras diferiram significativamente apenas para aroma alcoólico e gosto amargo. O efeito provador foi significativo para todos os atributos, no entanto não houve interação entre amostra e provador, indicando que todos os provadores julgaram as amostras da mesma maneira.

As médias dos valores hedônicos atribuídos pelos provadores a cada descritor são apresentadas na Tabela 3. Observou-se que a cachaça de kefir destacou-se dos demais atributos por apresentar um maior aroma alcoólico e gosto amargo. A cachaça de levedura caracterizou-se por apresentar uma maior ardência inicial.

Na Tabela 4 são apresentados os valores hedônicos médios de cada amostra para aceitação do aroma e para aceitação global, com os respectivos resultados do Teste de Tukey. Podese observar que não houve diferença significativa a $\mathrm{p} \leq 0,05$ entre nenhuma das amostras quanto à aceitação do aroma, indicando que as três cachaças foram igualmente aceitas pelos provadores quanto a esse atributo. Com relação à aceitação global, a amostra de levedura foi mais aceita dentre as três, atingindo valor hedônico próximo a 6 , correspondendo a gostei ligeiramente. No entanto, a simples média de aceitação, quando existem consumidores com preferências opostas, faz com que o resultado de alguns provadores anule o de outros, resultando em médias que podem não apresentar diferença significativa entre si.

Portanto para melhor visualizar possíveis diferenças na aceitação das amostras, foi construído um histograma da distribuição das frequências das respostas dos provadores para o aroma e aceitação global (Figuras 1 e 2). Na escala hedônica, a categoria "nem gostei, nem desgostei" (valor 5) é considerada como uma região de indiferença da relação afetiva do provador com o produto, dividindo a escala em duas outras regiões: a região de aceitação (valores de 6 a 9), e a região de rejeição do produto (valores de 1 a 4 ).

Com relação ao aroma, observa-se na Figura 1 que todas as amostras apresentaram os mais altos percentuais das respostas na região de aceitação da escala hedônica (valores de 6 a 9). As amostras desenvolvidas neste trabalho apresentaram distribuições muito semelhantes, em torno da moda 7, enquanto a amostra comercial apresentou moda 8 , sinalizando uma ligeira superioridade da aceitação do seu aroma.

Quanto à aceitação global, a amostra de levedura foi mais aceita dentre as três, atingindo valor hedônico próximo a gostei 
Tabela 1. Definições dos termos descritivos para a cachaça.

\begin{tabular}{|c|c|c|}
\hline Descritores & Definições & Amostras de referência \\
\hline Aroma alcoólico & Aroma característico de etanol que irrita a mucosa nasal. & $\begin{array}{l}\text { Fraco: Etanol 30\%. } \\
\text { Forte: Etanol 50\%. }\end{array}$ \\
\hline Aroma de cachaça & Aroma característico de cachaça comercial. & $\begin{array}{l}\text { Fraco: Etanol 20\%. } \\
\text { Forte: Cachaça Ypióca. }\end{array}$ \\
\hline Sabor alcoólico & Sabor característico de soluções alcoólicas. & $\begin{array}{l}\text { Fraco: Etanol 20\%. } \\
\text { Forte: Etanol 38\%. }\end{array}$ \\
\hline Sabor de cachaça & Sabor característico de cachaça comercial. & $\begin{array}{l}\text { Fraco: Etanol 20\%. } \\
\text { Forte: Cachaça Ypióca. }\end{array}$ \\
\hline Gosto doce & Gosto característico de sacarose. & $\begin{array}{l}\text { Fraco: Ausência. } \\
\text { Forte: Sol. } 0,5 \% \text { de sacarose em etanol 30\%. }\end{array}$ \\
\hline Gosto amargo & Gosto amargo característico de cafeína. & $\begin{array}{l}\text { Fraco: Etanol } 20 \% \text {. } \\
\text { Forte: Sol. } 0,05 \% \text { de cafeína em etanol } 20 \% \text {. }\end{array}$ \\
\hline Ardência inicial & Ardência inicial percebida na língua. & $\begin{array}{l}\text { Fraco: Etanol } 30 \% \\
\text { Forte: Etanol } 40 \%\end{array}$ \\
\hline
\end{tabular}

Tabela 2. Resultado da análise de variância dos descritores de cachaça para os efeitos Amostra (A), Provador $(\mathrm{P})$ e interação $\mathrm{A} \times \mathrm{P}$ (valores de $\mathrm{F}$ e níveis de significância).

\begin{tabular}{llcc}
\hline \multicolumn{1}{c}{ Atributo } & F amostra & F provador & F amostra $\times$ provador \\
\hline Aroma alcoólico & $5,50^{*}$ & $4,18^{* *}$ & 0,09 n.s. \\
Aroma cachaça & 0,10 n.s. & $6,74^{* *}$ & 1,14 n.s. \\
Sabor alcoólico & 0,00 n.s. & $9,77^{* *}$ & 1,35 n.s. \\
Gosto doce & 0,28 n.s. & $10,59^{* *}$ & 1,77 n.s. \\
Ardência inicial & 2,11 n.s. & $6,26^{* *}$ & 0,68 n.s. \\
Sabor de cachaça & 0,58 n.s. & $15,12^{* *}$ & 2,12 n.s. \\
Gosto amargo & $4,20^{*}$ & $8,74^{* *}$ & 1,51 n.s. \\
\hline n.s. = não significativo ao nível de $p<0,05$ de probabilidade; ${ }^{*}$ significativo ao nível $\mathrm{p}<0,05$ \\
de probabilidade; $\mathrm{e}^{* *}$ significativo ao nível $\mathrm{p}<0,01$ de probabilidade.
\end{tabular}

Tabela 3. Médias dos valores atribuídos pelos provadores a cada descritor, para as amostras de cachaça.

\begin{tabular}{lcc}
\hline \multicolumn{1}{c}{ Descritores } & \multicolumn{2}{c}{ Amostras } \\
\cline { 2 - 3 } & Levedura & Kefir \\
\hline Aroma alcoólico & $3,25^{\mathrm{b}}$ & $4,47^{\mathrm{a}}$ \\
Aroma cachaça & $3,71^{\mathrm{a}}$ & $3,85^{\mathrm{a}}$ \\
Sabor alcoólico & $4,38^{\mathrm{a}}$ & $4,39^{\mathrm{a}}$ \\
Gosto doce & $2,44^{\mathrm{a}}$ & $2,64^{\mathrm{a}}$ \\
Ardência inicial & $4,25^{\mathrm{a}}$ & $3,41^{\mathrm{a}}$ \\
Sabor de cachaça & $4,08^{\mathrm{a}}$ & $3,67^{\mathrm{a}}$ \\
Gosto amargo & $3,07^{\mathrm{b}}$ & $3,95^{\mathrm{a}}$ \\
\hline
\end{tabular}

Médias na mesma linha acompanhadas de mesma letra não diferem entre si ao nível de $5 \%$ de significância pelo teste de Tukey.

Tabela 4. Médias dos valores hedônicos para o aroma e aceitação global das amostras de cachaça em estudo e cachaça comercial.

\begin{tabular}{lcc}
\hline \multicolumn{1}{c}{ Amostras } & Aceitação do aroma & Aceitação global \\
\hline Cachaça de Kefir & $6,11^{\mathrm{a}}$ & $4,98^{\mathrm{b}}$ \\
Cachaça de S. cerevisae & $6,46^{\mathrm{a}}$ & $5,67^{\mathrm{a}}$ \\
Cachaça comercial & $6,32^{\mathrm{a}}$ & $4,49^{\mathrm{b}}$ \\
\hline
\end{tabular}

a,b Médias com mesma letra não diferem estatisticamente ao nível de 5\% de significância pelo teste de Tukey.

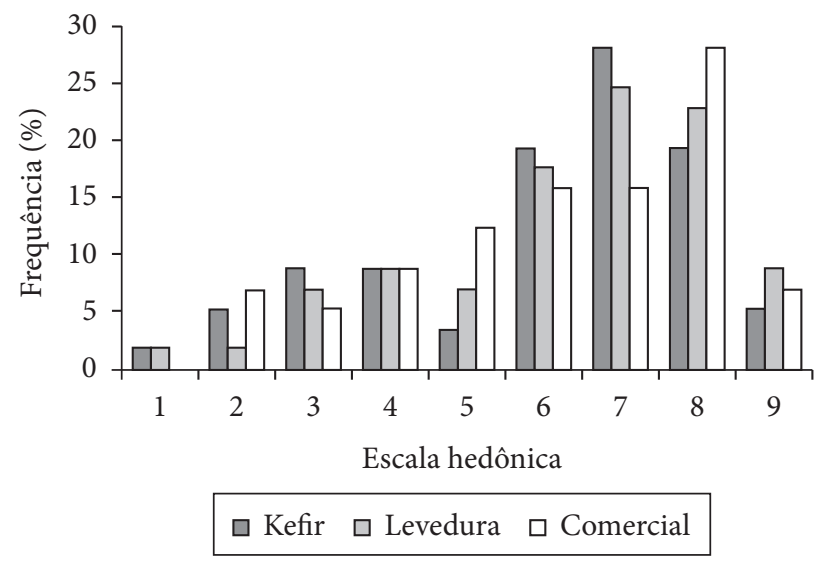

Figura 1. Histograma de frequência dos valores hedônicos atribuídos ao aroma das amostras de aguardente $(1=$ desgostei muitíssimo, $5=$ nem gostei, nem desgostei, 9 = gostei muitíssimo).

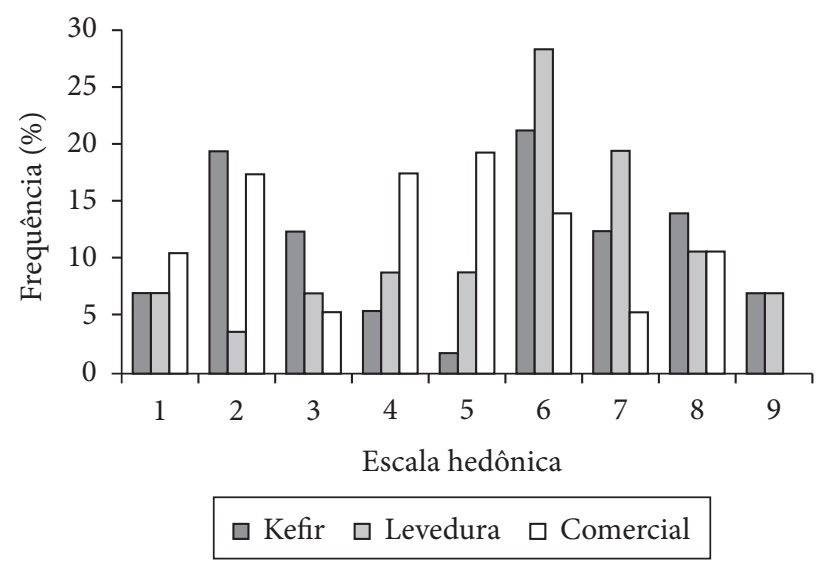

Figura 2. Histograma de frequência dos valores hedônicos atribuídos à aceitação global das amostras de aguardente ( 1 = desgostei muitíssimo, $5=$ nem gostei, nem desgostei 9 = gostei muitíssimo).

ligeiramente (6) como podemos observar na Tabela 4. A amostra utilizando os grânulos de Kefir ficou na região de indiferença com valor hedônico próximo a 5, correspondente a "nem gostei 
e nem desgostei". A amostra comercial encontrou-se abaixo da região de indiferença, tendendo para a região de rejeição.

Analisando-se a Figura 2, observa-se que a distribuição da frequência dos valores hedônicos de aceitação global atribuídos à cachaça elaborada com levedura ficou deslocada para a região de aceitação, de forma monomodal, em torno da moda 6 (gostei ligeiramente), enquanto que as distribuições das outras amostras apresentaram-se bimodais, ou seja, em torno de duas modas. A amostra de kefir apresentou um pico de frequência no valor 2 (rejeição) e outro em torno de 6 (aceitação), indicando que uma parte dos consumidores gostou da amostra, mas outro grupo não gostou.

O mesmo fato foi observado para a amostra comercial, porém com modas nos valores 2 e 5 , indicando que essa amostra apresentou menor aceitação global que as demais.

Para facilitar a interpretação desses resultados, foram somadas as frequências das categorias da região de rejeição, indiferença e aceitação, construindo-se novas variáveis que foram chamadas de percentual de rejeição $(\% \mathrm{R})$, percentual indiferença (\%I) e percentual de aprovação (\%A), respectivamente, as quais são apresentadas na Figura 3. Nessa Figura fica clara a segmentação dos provadores em dois grupos quanto à amostra fermentada com kefir, sendo as respostas de aceitação e rejeição praticamente divididas meio a meio (foi mínima a porcentagem de respostas na categoria de indiferença).

A amostra com Kefir apresentou menor aceitação (54,39\%) quando comparada à amostra de cachaça produzida com levedura $(64,91 \%)$, porém sua aceitação pelos consumidores foi bem maior que a da amostra comercial, a qual apresentou (50,88\%) de rejeição, (19,30\%) de indiferença e $(29,82 \%)$ de aprovação.

Esse desempenho da amostra fermentada com kefir mostra um grande potencial para esse produto, visto que ele apresentou melhor aceitabilidade que o produto comercial, o qual foi escolhido entre as marcas líderes de mercado na região.

A cachaça de levedura continha um teor de açúcar de 4 g.L $L^{-1}$, concentração permitida pela legislação (BRASIL, 2005), enquanto à cachaça de Kefir não foi adicionada sacarose para

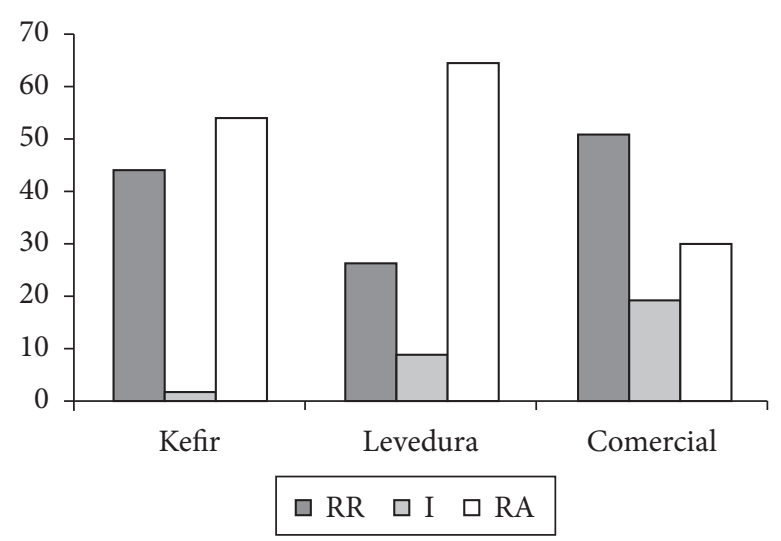

Figura 3. Porcentagens de aprovação, indiferença e rejeição da aceitação global das amostras de aguardente. RR: região de rejeição; I: região de indiferença; RA: região de aceitação. não mascarar o seu sabor original. Dessa forma acredita-se que a cachaça de Kefir poderia ter sido mais aceita, caso tivesse um grau de doçura maior.

\section{Conclusões}

O estudo da aceitação da cachaça produzida com grânulos de Kefir mostrou que o seu grau de aceitação foi satisfatório, no entanto o resultado de satisfação para a cachaça obtida com levedura S. cerevisae foi melhor. Podemos atribuir essa menor aceitação da cachaça produzida com Kefir por esta ser um produto novo com sabor diferenciado do produto tradicional. $\mathrm{O}$ estudo do perfil da cachaça com Kefir apresentou maior intensidade de aroma alcoólico e gosto amargo, obtendo menor aceitação global que a cachaça de levedura. Entretanto o produto foi considerado de grande potencial visto que apresentou um bom percentual de aprovação entre os provadores e melhores resultados podem ser obtidos através do envelhecimento da cachaça de Kefir, que é objeto de trabalhos futuros.

\section{Referências bibliográficas}

AQUINO, F. W. B. et al. Determinação de marcadores de envelhecimento em cachaças. Ciências e Tecnologia de Alimentos, v. 26, n. 1, p. $145-149$ p, 2006.

BOZA, Y.; HORII, J. Influência da destilação sobre a composição e a qualidade sensorial da aguardente de cana-de-açúcar. Ciências e Tecnologia de Alimentos, v. 18, n. 4, p. 391-396, 1998.

BRASIL, Ministério da Agricultura. Regulamento Técnico para Fixação dos Padrões de Identidade e Qualidade para Aguardente de Cana e para Cachaça. Instrução Normativa, n. 13 de 29 de junho de 2005. Diário Oficial da União, 30 de Junho de 2005, Seção 1, Página 3.

CARDELLO, H. M. A. B.; FARIA, J. B. Análise descritiva quantitativa da aguardente de cana durante o envelhecimento em tonel de carvalho (Quercus Alba L.). Ciência e Tecnologia de Alimentos, v. 18, n. 2, Maio/Julho, 1998.

DORNELLES, A. S.; RODRIGUES, S. Fermentação Alcoólica de Caldo de Cana utilizando grãos de Kefir. Ciência Agronômica, v. 37, n. 3, p. 386-390, 2006.

DORNELLES, A. S. Produção de cachaça com grânulos de Kefir. Fortaleza, 2007. 72 f. Dissertação (Mestrado em Tecnologia de Alimentos) - Departamento de Tecnologia de Alimentos, Universidade Federal do Ceará - UFC.

GARROTE, G. L. et al. Rapid discrimination of lactobacilli isolated from kefir grains by FT-IR spectroscopy. International Journal of Food Microbiology, v. 111, n. 3, p. 280-287, 2006.

JANZANTTI, N. S. Compostos Voláteis e qualidade de sabor de cachaça. Campinas, 2004. Tese (Doutorado em Ciência de Alimentos) - Faculdade de Engenharia de Alimentos, Universidade Estadual de Campinas.

Jeronimo, E. M.; CARDEllo, H. M. A. B.; SERRA, G. E. Perfil sensorial de aguardente de cana em função da diluição e variação da acidez da amostra. Boletim do Centro de Pesquisa e Processamento de Alimentos, v. 22, n. 1, p. 51-64, 2004.

MEILGAARD, M.; CIVILLE, G. V.; CARR, B. T. Sensory Evaluation Techniques. 1ed. Florida, USA: CRC Press, 1987. p. 354.

STATYSTICAL ANALISYS SISTEM. SAS/STAT: Users guide. Version 6. 12 ed. Cary: Sas Institute Inc., 2000.

STONE, H. et al. Sensory evaluation by quantitative descriptive analysis. Food Technology, v. 28, p. 24-34, 1974. 